\title{
Electromagnetic Resonances of a Straight Wire
}

\section{Citation}

Myers, John M., Sheldon S. Sandler, and Tai Tsun Wu. 2011. "Electromagnetic Resonances of a Straight Wire." IEEE Trans. Antennas Propagat. 59, no. 1: 129-134.

\section{Published Version}

doi:10.1109/tap.2010.2090479

\section{Permanent link}

http://nrs.harvard.edu/urn-3:HUL.InstRepos:12701525

\section{Terms of Use}

This article was downloaded from Harvard University's DASH repository, and is made available under the terms and conditions applicable to Open Access Policy Articles, as set forth at http:// nrs.harvard.edu/urn-3:HUL.InstRepos:dash.current.terms-of-use\#OAP

\section{Share Your Story}

The Harvard community has made this article openly available.

Please share how this access benefits you. Submit a story.

\section{Accessibility}




\title{
Electromagnetic Resonances of a Straight Wire
}

\author{
John M. Myers, Sheldon S. Sandler, and Tai Tsun Wu
}

\begin{abstract}
With an interest in finding wires and distinguishing them from other electrically conducting objects, we have looked for an electromagnetic "fingerprint" in terms of resonances of a straight wire of length $2 h$ and radius $a$. The resonances of the wire are formulated using the theory of the linear antenna, leading to an integral equation for the current on the wire. Complexvalued resonant frequencies are defined as those for which the homogeneous integral equation for the current on the wire has non-zero solutions. By applying a variational technique we obtain approximate numerical solutions for the resonant frequencies and their widths. A table of the first five resonances is given for several ratios of wire half-length $h$ to wire radius $a$. In a subsequent paper we propose to extend the method described here to deal with wires on an earth-air interface, for example as used to command the detonation of improvised explosive devices.
\end{abstract}

\section{Index Terms}

Antenna theory, integral equation, resonance, variational methods.

\section{INTRODUCTION}

W $\mathrm{E}$ are interested in the backscattering properties of a straight wire, and in particular in the first five resonant frequencies that characterize a wire as distinct from other conducting objects. Although studied for over a century, properties of the electromagnetic field associated with a straight wire in free space remain a challenge to determine. The dipole antenna consisting of a straight, perfectly conducting wire driven at its center by an applied voltage has been studied, and the first two resonant frequencies have been determined, based on approximate solutions to an integral equation [1]; however, the problem of resonant frequencies for a very thin wire driven off center has eluded an accurate solution. A related problem of the scattering of electromagnetic energy by a fat wire was analyzed [2] using the Singularity Expansion Method [3]; however, again the scattering by a very thin sourceless wire has also eluded accurate solution, in part for the same reason, namely that neither analytic nor numerical methods suffice to obtain accurate solutions to the integral equation for the current in the thin wire. For numerical work, the difficulty is that the grid spacing for approximating the current must be very fine, resulting in the need to invert $N$-by- $N$ matrices with $N$ on the order of the ratio of wire length to wire diameter. For command wires, this means $N \geq 10^{4}$. While the center-driven antenna exhibits a current density symmetric about the driving point, both the unsymmetrically driven antenna and the sourceless wire as a scattering object involve resonances for which the current is antisymmetric about the center of the wire.

In this paper we formulate the problem of determining resonant frequencies of a straight, perfectly conducting wire as a Pocklington integral equation for the current in the wire. As in the Singularity Expansion Method [3], we define both the resonant frequencies and the widths of these resonances in terms of the complex-valued frequencies at which the homogeneous Pocklington equation has non-zero solutions. We then determine these complex frequencies approximately, using a variational technique, for both the cases in which the current along the thin wire is symmetric about the center point and

This work was supported in part by the Army Research Office under Grant W911NF-07-1-0509 with Harvard University. The authors are with the Harvard School of Engineering and Applied Sciences, Cambridge, MA 02138 USA. (e-mail: myers@seas.harvard.edu; sandler@seas.harvard.edu; ttwu@seas.harvard.edu.) 
for the antisymmetric cases, which, so far as we know, have not previously been found. The important advantage of the variational technique is its relative insensitivity to errors in the current, allowing a rough approximation to the resonant currents to be used to obtain a close approximation to resonant frequencies and the widths of the resonances.

\section{FORMULATION}

We represent a straight wire subject to electromagnetic illumination by a thin, perfectly conducting cylinder of radius $a$ and length $2 h$, embedded in an infinite uniform lossless medium with dielectric constant $\epsilon$ and magnetic permeability $\mu_{0}$. The axis of the wire coincides with the $x$-axis of a coordinate system and we consider an incident field at a single angular frequency $\omega$, which induces a spatially varying current $I(x)$ along the wire. This is defined by:

$$
\left(\frac{\partial^{2}}{\partial x^{2}}+k^{2}\right) \int_{-h}^{h} d x^{\prime} K\left(x-x^{\prime}\right) I\left(x^{\prime}\right)=4 \pi i k \sqrt{\mu_{0} / \epsilon_{0}} E_{x}(x)
$$

along with the boundary condition that $I( \pm h)=0$. In (1), $E(x)$ is the $x$-component of the incident electric field, $k=\omega / c$ (with $c$ the speed of light) is the propagation constant, and the kernel $K$ is defined in Appendix A as a function of $x$ and $k$ by

$$
K(x)=\frac{1}{2 \pi} \int_{0}^{2 \pi} d \theta \frac{\exp \left(i k \sqrt{2 a^{2}(1-\cos \theta)+x^{2}}\right)}{\sqrt{2 a^{2}(1-\cos \theta)+x^{2}}} .
$$

If there is no incident field, that is, if $E_{x}(x)=0$ on $-h \leq x \leq h$, it follows that (1) specializes to

$$
\left(\frac{\partial^{2}}{\partial x^{2}}+k^{2}\right) \int_{-h}^{h} d x^{\prime} K\left(x-x^{\prime}\right) I\left(x^{\prime}\right)=0 .
$$

At first glance, one might expect the only solution to be $I(x)=0$. However, the key to defining resonance is to note that the kernel $K$ depends not only on position along the wire, but also on the frequency $\omega=c k$. Therefore the solution to the integral equation (3) depends on $\omega$ and for certain discrete special values of $\omega, \omega_{1}=c k_{1}, \omega_{2}=c k_{2}, \ldots$, the equation has non-zero solutions. These values are expected to be complex, so that, listed in increasing order of their real parts, we have

$$
\begin{aligned}
\operatorname{Re} \omega_{n} & =\text { the } n \text {-th resonant frequency, } \\
-\operatorname{Im} \omega_{n} & =\text { the half-width at half-maximum of the } n \text {-th resonance. }
\end{aligned}
$$

We assume that the resonant currents are proportional to $1 /\left(\omega-\omega_{n}\right)$. The issue is how to find the $\omega_{n}$, or equivalently the $k_{n}$, at which (3) has non-zero solutions.

\section{APPROXIMATE SOLUTION FOR RESONANT FREQUENCIES}

Symbolically, we let $A(k)$ denote the linear operator in (3), so that the equation is abbreviated as $A(k) * I_{n}(k)=0$, where $*$ denotes an integral over the spatial variable, and we have omitted writing the spatial variables $x$ and $x^{\prime}$ while we have made explicit the dependence of $A$ and $I_{n}$ on the propagation constant $k$. For a thin wire, it is known that the resonant frequencies correspond to $k_{n}$ near $n \pi / 2 h$. Our problem of resonance is to determine for $n=1, \ldots, 5$ the $k_{n}$ near $n \pi / 2 h$ such that $A\left(k_{n}\right) * I_{n}\left(k_{n}\right)=0$ has a solution for non-zero $I_{n}\left(k_{n}\right)$. What we require is only $k_{n}$; we do not seek an accurate solution to the current $I_{n}(k) \equiv I_{n}(k, x)$.

To find $k_{n}$, we start by considering the functional

$$
\begin{aligned}
S[I] & \stackrel{\text { def }}{=} I(k) * A(k) * I(k) \\
& =\int_{-h}^{h} d x I(k, x)\left(\frac{d^{2}}{d x^{2}}+k^{2}\right) \int_{-h}^{h} d x^{\prime} K\left(x-x^{\prime}\right) I\left(k, x^{\prime}\right) .
\end{aligned}
$$


Suppose that for some value $k_{n}$ there is a non-zero solution $I_{n}\left(k_{n}, x\right)$ to (3). As shown in Appendix $\mathrm{B}$, the variation with respect to $I$ (as a function of $x$ ) around this $I_{n}\left(k_{n}\right)$ of $S[I]$ is zero; that is: $0=$ $\delta\left[I_{n}\left(k_{n}\right) * A\left(k_{n}\right) * I_{n}\left(k_{n}\right)\right]$. Equation (3) is just the statement that $A(k) * I_{n}(k)=0$, whence it follows that $I_{n}\left(k_{n}\right) * A\left(k_{n}\right) * I_{n}\left(k_{n}\right)=0$. But since the first variation of the left-hand side is zero, replacing $I_{n}\left(k_{n}\right)$ by an approximation $I_{n}^{\text {ap }}$ makes no first-order error in the expression $I_{n}^{\text {ap }} * A\left(k_{n}\right) * I_{n}^{\text {ap }}$. Thus we will determine $k_{n}$ as the solution, for a suitable approximating current $I_{n}^{\text {ap }}$, to

$$
0=I_{n}^{\mathrm{ap}} * A\left(k_{n}\right) * I_{n}^{\mathrm{ap}} .
$$

For computational convenience we carry an $x$-derivative under the integral, note that $d K\left(x-x^{\prime}\right) / d x=$ $-d K\left(x-x^{\prime}\right) / d x^{\prime}$, and integrate by parts to obtain for the equation for $k_{n}$

$$
0=\int_{-h}^{h} d x \int_{-h}^{h} d x^{\prime} \frac{d I_{n}^{\mathrm{ap}}(x)}{d x} K\left(x-x^{\prime}\right) \frac{d I_{n}^{\mathrm{ap}}\left(x^{\prime}\right)}{d x^{\prime}}-k^{2} \int_{-h}^{h} d x \int_{-h}^{h} d x^{\prime} I_{n}^{\mathrm{ap}}(x) K\left(x-x^{\prime}\right) I_{n}^{\mathrm{ap}}\left(x^{\prime}\right) .
$$

The dependence on $k_{n}$, the sought value of $k$, is now limited to the dependence of the kernel $K\left(x-x^{\prime}\right)$ on $k$ as expressed in (2).

Now we choose an approximating current. As noted above, for sufficiently small $a$ one expects resonant frequencies at values of $k$ near

$$
\kappa_{n} \stackrel{\text { def }}{=} \frac{n \pi}{2 h}
$$

for $n=1,2, \ldots$, with the currents corresponding to odd values of $n$ symmetric in $x$ while the currents corresponding to even values of $n$ are antisymmetric in $x$ [2].

For the symmetric case, there are good reasons to believe that the resonance current is given roughly by the shifted-cosine form $\cos k x-\cos k h$ [1]. One might expect to get a good approximation to the complex resonant frequency by choosing the current to be the shifted cosine; however, use of the shifted cosine can result in possibly spurious values of the resonant frequency. Examination of how these values arise shows that they are indeed spurious artifacts of the shifted-cosine form; hence we need to attend more carefully to the choice of the approximating current. The shifted-cosine form leads to an approximating current dependent on the frequency $\omega \sim k$. Question: should the approximating current depend on the frequency $\omega$ or not? Having encountered spurious values from the shifted-cosine form, we choose instead an approximating current that is independent of the frequency $\omega$. This independence essentially determines the approximation. To begin with, it can depend only on the geometric parameters $h$ and $a$. Although we could force the approximation to depend on $a$, we cannot see how to do this in a physically sensible way. Thus we take the approximation to the resonant current to depend only on $h$. Then we can hardly avoid choosing $I_{n}^{\text {ap }} \sim \cos \kappa_{n} x=\cos (n \pi x / 2 h)$ for the symmetric case where $n$ is odd. Correspondingly, for the cases of resonance in which the current is antisymmetric about the center point of the wire, for which $n$ is even, our approximation to the resonant current is $I_{n}^{\text {ap }} \sim \sin \kappa_{n} x$. We expect these approximate currents to be adequate for use in (7) for the first five resonant frequencies, but not for much higher resonances.

\section{A. Resonances}

With the chosen approximating currents, (7) for determining $k_{n}$ becomes

(For $n$ odd)

$$
\begin{aligned}
& 0=\kappa_{n}^{2} \int_{-h}^{h} d x \sin \frac{n \pi x}{2 h} \int_{-h}^{h} d x^{\prime} K\left(x-x^{\prime}\right) \sin \frac{n \pi x^{\prime}}{2 h} \\
& -k^{2} \int_{-h}^{h} d x \cos \frac{n \pi x}{2 h} \int_{-h}^{h} d x^{\prime} K\left(x-x^{\prime}\right) \cos \frac{n \pi x^{\prime}}{2 h} ; \\
& \text { (For } n \text { even) } \quad 0=\kappa_{n}^{2} \int_{-h}^{h} d x \cos \frac{n \pi x}{2 h} \int_{-h}^{h} d x^{\prime} K\left(x-x^{\prime}\right) \cos \frac{n \pi x^{\prime}}{2 h} \\
& -k^{2} \int_{-h}^{h} d x \sin \frac{n \pi x}{2 h} \int_{-h}^{h} d x^{\prime} K\left(x-x^{\prime}\right) \sin \frac{n \pi x^{\prime}}{2 h} \text {. }
\end{aligned}
$$

Each of this pair of expressions involves the same two integrals 


$$
\begin{gathered}
I_{s} \stackrel{\text { def }}{=} \int_{-h}^{h} d x \sin \kappa x \int_{-h}^{h} d x^{\prime} K\left(x-x^{\prime}\right) \sin \kappa x, \\
I_{c} \stackrel{\text { def }}{=} \int_{-h}^{h} d x \cos \kappa x \int_{-h}^{h} d x^{\prime} K\left(x-x^{\prime}\right) \cos \kappa x .
\end{gathered}
$$

In terms of these integrals, (9) and (10) can be put in a form convenient for calculating $k_{n}$ :

$$
\begin{aligned}
& \left(\kappa_{n}^{2}-k^{2}\right)\left(I_{s}+I_{c}\right)+\left(\kappa_{n}^{2}+k^{2}\right)\left(I_{s}-I_{c}\right)=0, \quad n \text { odd } \\
& \left(\kappa_{n}^{2}-k^{2}\right)\left(I_{s}+I_{c}\right)-\left(\kappa_{n}^{2}+k^{2}\right)\left(I_{s}-I_{c}\right)=0, \quad n \text { even. }
\end{aligned}
$$

We now make a zero-th order check. (Given any positive numbers $\epsilon$ and $\eta$ and a wire of half-length $h$, there is some $a_{0}$, depending on $\epsilon$ and $\eta$, such that for $a<a_{0}$ the kernel in the integral in (3) acts essentially as a delta-function. More precisely the integral of the absolute value of the kernel over the integration range $2 h>\left|x-x^{\prime}\right|>\epsilon$ can be made less than $\eta$ times the integral of the absolute value of the kernel over the small integration range $\left|x-x^{\prime}\right| \leq \epsilon$.) Upon replacing $K\left(x-x^{\prime}\right)$ by a delta function $\delta\left(x-x^{\prime}\right)$, carrying out the integrals in (9), and using the definition of $\kappa_{n}=n \pi / 2 h$, the result for $n$ odd is

$$
-\kappa_{n}^{2}+k^{2}=0 \text {. }
$$

Carrying out the same procedure on (10) yields this relation for $n$ even-confirming our claim that for sufficiently small radius, the resonant propagation constants should be near $\kappa_{n}$.

Returning to the use of $K\left(x-x^{\prime}\right)$ and not just the delta function, we want to hold fixed the geometrical and material parameters $h, a, \epsilon$, and $\mu_{0}$ and vary only the propagation constant $k=\omega / c$, which amounts to varying the frequency $\omega$ in order to find solutions to (13) for the symmetric resonances and (14) for the antisymmetric resonances. The first step, derived in Appendix C, is to reduce the double integrals to single integrals to obtain:

$$
\begin{aligned}
& I_{s}+I_{c}=2 \int_{0}^{2 h} d y K(y)(2 h-y) \cos \kappa_{n} y, \\
& I_{s}-I_{c}=-\frac{2}{\kappa_{n}} \int_{0}^{2 h} d y K(y) \sin \left[\kappa_{n}(2 h-y)\right] .
\end{aligned}
$$

When the wire radius $a$ is much smaller than all the other dimensions in this scattering problem, we can approximate the kernel $K$ defined in (2). For $x \gg a$, the kernel is very close to

$$
K(y) \approx \frac{e^{i k \sqrt{y^{2}+a^{2}}}}{\sqrt{y^{2}+a^{2}}},
$$

and furthermore the integral over the logarithmic singularity in $K$ is closely matched by the integral over the approximation defined in (17). Note that $k$ is complex. With this approximation one obtains from (16) and the definition of $\kappa_{n}$ in (8)

$$
\begin{aligned}
I_{s}-I_{c} & =-\frac{2}{\kappa_{n}} \int_{0}^{2 h} d y \frac{e^{i k \sqrt{y^{2}+a^{2}}}}{\sqrt{y^{2}+a^{2}}} \sin \left[\kappa_{n}(2 h-y)\right] \\
& =(-1)^{n} \frac{2}{\kappa_{n}} \int_{0}^{2 h} d y \frac{e^{i k \sqrt{y^{2}+a^{2}}}}{\sqrt{y^{2}+a^{2}}} \sin \kappa_{n} y \approx(-1)^{n} \frac{2}{\kappa_{n}} \int_{0}^{2 h} d y \frac{e^{i k y}}{y} \sin \kappa_{n} y,
\end{aligned}
$$

where the last approximation makes negligible error when $a$ is much smaller than the other dimensions. It will turn out that the resonant frequencies correspond to $\operatorname{Im} k<0$, so that the integrand in (18) is an increasing function of $y$. As evaluated in Appendix D, we obtain

$$
\begin{aligned}
I_{s}+I_{c}= & 4 h\left[\ln \frac{4 h}{a}-\int_{0}^{2 h} \frac{d y}{y}\left(1-e^{i k y} \cos \kappa_{n} y\right)\right] \\
& +\frac{i}{k+\kappa_{n}}\left[e^{i 2\left(k+\kappa_{n}\right) h}-1\right]+\frac{i}{k-\kappa_{n}}\left[e^{i 2\left(k-\kappa_{n}\right) h}-1\right] .
\end{aligned}
$$


TABLE I

COMPlex VAlues of $k h$ AT Resonance $n$ (Where $\kappa_{n}=n \pi / 2 h$ )

\begin{tabular}{|c|c|c|c|c|c|}
\hline$n$ & $h / a=10^{4}$ & $n$ & $h / a=10^{5}$ & $n$ & $h / a=10^{6}$ \\
\hline 1 & $1.522174-i 0.066372$ & 1 & $1.533583-i 0.053232$ & 1 & $1.540710-i 0.044393$ \\
\hline 2 & $3.086149-i 0.089877$ & 2 & $3.099893-i 0.071216$ & 2 & $3.108258-i 0.058920$ \\
\hline 3 & $4.653231-i 0.104815$ & 3 & $4.668363-i 0.082482$ & 3 & $4.677431-i 0.067934$ \\
\hline 4 & $6.221436-i 0.115911$ & 4 & $6.237582-i 0.090775$ & 4 & $6.247152-i 0.074530$ \\
\hline 5 & $7.790220-i 0.124802$ & 5 & $7.807177-i 0.097374$ & 5 & $7.817142-i 0.079757$ \\
\hline
\end{tabular}

Putting all this together, we need to solve numerically for complex $k$ the equation

$$
\begin{aligned}
\left(\kappa_{n}^{2}-k^{2}\right)\{ & 4 h\left[\ln \frac{4 h}{a}-\int_{0}^{2 h} \frac{d y}{y}\left(1-e^{i k y} \cos \kappa_{n} y\right)\right] \\
& \left.+\frac{i}{k+\kappa_{n}}\left[e^{i 2\left(k+\kappa_{n}\right) h}-1\right]+\frac{i}{k-\kappa_{n}}\left[e^{i 2\left(k-\kappa_{n}\right) h}-1\right]\right\} \\
& -\left(\kappa_{n}^{2}+k^{2}\right) \frac{2}{\kappa_{n}} \int_{0}^{2 h} d y \frac{e^{i k y}}{y} \sin \kappa_{n} y=0
\end{aligned}
$$

Numerical analysis then yields the examples shown in Table I. Figure 1 displays these data graphically.

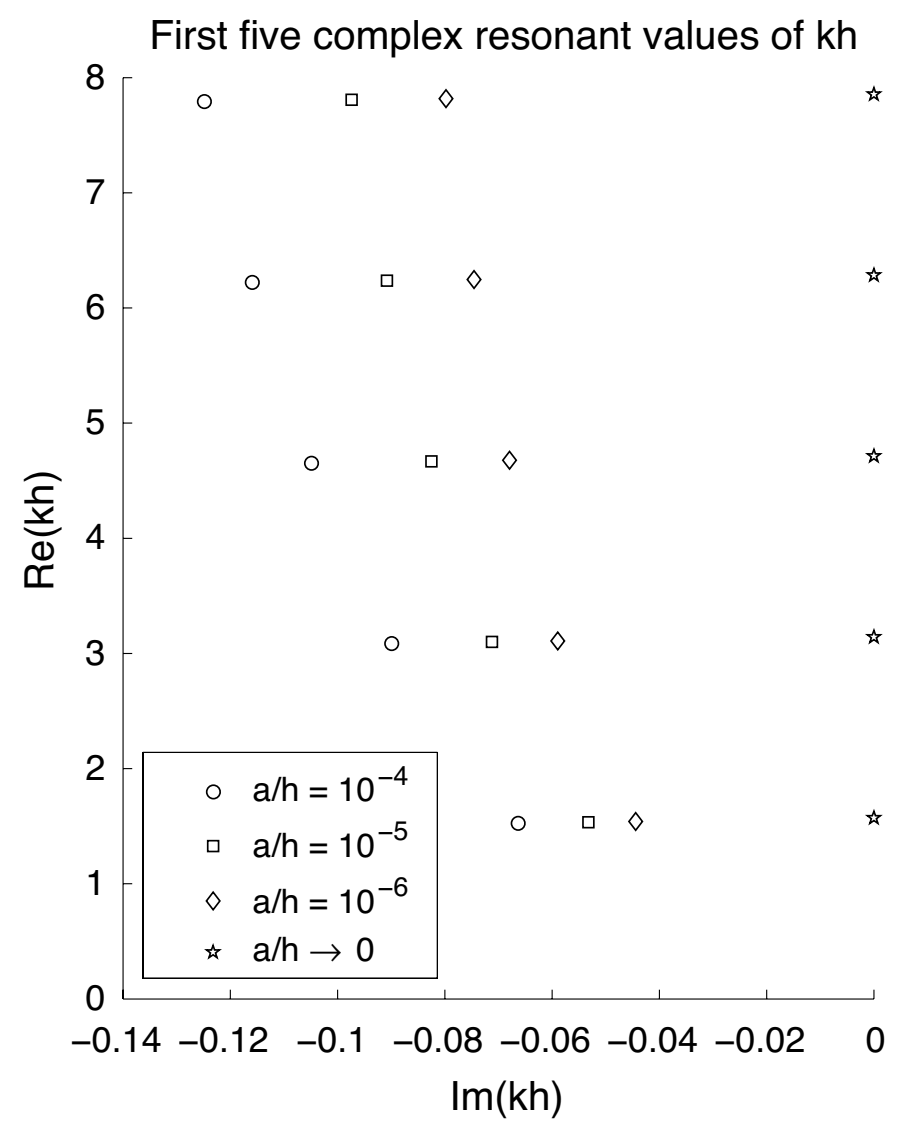

Fig. 1. Complex resonant values on the $k h$-plane. 


\section{Discussion}

Naively, one pictures a resonance as a large response to a small incident field. Thought of this way, the calculation of resonance seems to demand choosing one or more incident fields, and the choice of these fields defies any simple physical basis. For example, one might consider plane waves or one might consider an incident field generated by one or another transmitting antenna located at some distance and orientation from the scattering wire. Except for the case of an incident field transverse to the wire, for which very little scattering occurs, one expects the resonant frequencies to be largely insensitive to the incident field. Here we have taken advantage of the near-independence of resonances from the choice of incident field to define resonances in terms of non-zero solutions to the homogeneous Pocklington equation.

The other noteworthy feature is to make use of the variational technique to show that the complex resonant frequencies are insensitive to small errors in the resonant current, which justifies replacing the resonant current, which is exceedingly difficult to determine to high accuracy, by an approximate current, as discussed above.

\section{APPENDIX A}

\section{KERNEL}

The kernel $K$ in (1) is defined as a function of $x$ and $k$ by the Green's function as follows. Consider cylindrical coordinates $(\rho, \phi, x)$ oriented around the $x$-axis, so that the distance to a point from the $x$-axis is given by $\rho=\sqrt{y^{2}+z^{2}}$; correspondingly we have the cartesian components $y=\rho \cos \phi, z=\rho \sin \phi$. The retarded Green's function defines the vector potential on the wire surface at a point $(a, \phi, x)$ arising from a current density $\boldsymbol{J}\left(a, \phi^{\prime}, x^{\prime}\right)$ on the wire by

$$
\boldsymbol{A}(a, \phi, x)=\frac{\mu_{0}}{4 \pi} \int_{-h}^{h} d x^{\prime} \int_{0}^{2 \pi} a d \phi^{\prime} \frac{\exp \left(i k \sqrt{2 a^{2}\left[1-\cos \left(\phi-\phi^{\prime}\right)\right]+\left(x-x^{\prime}\right)^{2}}\right)}{\sqrt{2 a^{2}\left[1-\cos \left(\phi-\phi^{\prime}\right)\right]+\left(x-x^{\prime}\right)^{2}}} \boldsymbol{J}\left(x^{\prime}\right) .
$$

We now average over $\phi$ to obtain

$$
\begin{aligned}
\frac{1}{2 \pi} & \int_{0}^{2 \pi} d \phi \boldsymbol{A}(a, \phi, x) \\
& =\frac{\mu_{0}}{4 \pi} \int_{-h}^{h} d x^{\prime} \int_{0}^{2 \pi} a d \phi^{\prime} \boldsymbol{J}\left(x^{\prime}\right) \frac{1}{2 \pi} \int_{0}^{2 \pi} d \phi \frac{\exp \left(i k \sqrt{2 a^{2}\left[1-\cos \left(\phi-\phi^{\prime}\right)\right]+\left(x-x^{\prime}\right)^{2}}\right)}{\sqrt{2 a^{2}\left[1-\cos \left(\phi-\phi^{\prime}\right)\right]+\left(x-x^{\prime}\right)^{2}}} \\
& =\frac{\mu_{0}}{4 \pi} \int_{-h}^{h} d x^{\prime} \int_{0}^{2 \pi} a d \phi^{\prime} \boldsymbol{J}\left(x^{\prime}\right) \frac{1}{2 \pi} \int_{0}^{2 \pi} d \theta \frac{\exp \left(i k \sqrt{2 a^{2}(1-\cos \theta)+\left(x-x^{\prime}\right)^{2}}\right)}{\sqrt{2 a^{2}(1-\cos \theta)+\left(x-x^{\prime}\right)^{2}}} \\
& =\frac{\mu_{0}}{4 \pi} \int_{-h}^{h} d x^{\prime} K\left(x-x^{\prime}\right) \boldsymbol{I}\left(x^{\prime}\right),
\end{aligned}
$$

where we define

$$
\begin{aligned}
\boldsymbol{I}\left(x^{\prime}\right) & =\int_{0}^{2 \pi} a d \phi^{\prime} \boldsymbol{J}\left(a, \phi^{\prime}, x^{\prime}\right), \\
K(x) & =\frac{1}{2 \pi} \int_{0}^{2 \pi} d \theta \frac{\exp \left(i k \sqrt{2 a^{2}(1-\cos \theta)+x^{2}}\right)}{\sqrt{2 a^{2}(1-\cos \theta)+x^{2}}} .
\end{aligned}
$$

The kernel $K(x)$ enters both the Hallén integral equation [1] and the Pocklington equation. For a wire with $k a \ll 1$, we assume that only the $x$-components of the current density and of the vector potential are relevant, and (1) follows. 


\section{APPENDIX B}

\section{VARIATIONAL FORM OF INTEGRAL EQUATION}

We study the variational of $S[I]$ defined in (5) with respect to $I(k, x)$, with $k$ fixed. Computing the variation one finds

$$
\begin{aligned}
\delta S[I]= & \int_{-h}^{h} d x[\delta I(k, x)]\left(\frac{d^{2}}{d x^{2}}+k^{2}\right) \int_{-h}^{h} d x^{\prime} K\left(x-x^{\prime}\right) I\left(k, x^{\prime}\right) \\
& +\int_{-h}^{h} d x I(k, x) \frac{d^{2}}{d x^{2}} \int_{-h}^{h} d x^{\prime} K\left(x-x^{\prime}\right) \delta I\left(k, x^{\prime}\right) \\
& +k^{2} \int_{-h}^{h} d x I(k, x) \int_{-h}^{h} d x^{\prime} K\left(x-x^{\prime}\right) \delta I\left(k, x^{\prime}\right) .
\end{aligned}
$$

Denote the second term by $T_{2}$ and invoke the boundary condition $I(k, x) \rightarrow 0$ as $x \rightarrow \pm h$ to obtain, dropping the explicit mention of $k$,

$$
\begin{aligned}
T_{2} & \stackrel{\text { def }}{=} \int_{-h}^{h} d x I(x) \frac{d^{2}}{d x^{2}} \int_{-h}^{h} d x^{\prime} K\left(x-x^{\prime}\right) \delta I\left(x^{\prime}\right) \\
& =-\int_{-h}^{h} d x I(x) \frac{\partial}{\partial x} \int_{-h}^{h} d x^{\prime} \frac{\partial K\left(x-x^{\prime}\right)}{\partial x^{\prime}} \delta I\left(x^{\prime}\right) \\
& =\int_{-h}^{h} d x \frac{d I(x)}{d x} \int_{-h}^{h} d x^{\prime} \frac{\partial K\left(x-x^{\prime}\right)}{\partial x^{\prime}} \delta I\left(x^{\prime}\right) \\
& =\int_{-h}^{h} d x^{\prime} \frac{d I\left(x^{\prime}\right)}{d x^{\prime}} \int_{-h}^{h} d x \frac{\partial K\left(x-x^{\prime}\right)}{\partial x} \delta I(x) \\
& =\int_{-h}^{h} d x \int_{-h}^{h} d x^{\prime}[\delta I(x)] \frac{\partial K\left(x-x^{\prime}\right)}{\partial x} \frac{d I\left(x^{\prime}\right)}{d x^{\prime}} \\
& =\int_{-h}^{h} d x[\delta I(x)] \int_{-h}^{h} d x^{\prime} \frac{\partial K\left(x-x^{\prime}\right)}{d I\left(x^{\prime}\right)} \\
& =\int_{-h}^{h} d x[\delta I(x)] \frac{d}{d x} \int_{-h}^{h} d x^{\prime} K\left(x-x^{\prime}\right) \frac{d I\left(x^{\prime}\right)}{d x^{\prime}} \\
& =-\int_{-h}^{h} d x[\delta I(x)] \frac{d}{d x} \int_{-h}^{h} d x^{\prime} \frac{\partial K\left(x-x^{\prime}\right)}{\partial x^{\prime}} I\left(x^{\prime}\right) \\
& =\int_{-h}^{h} d x[\delta I(x)] \frac{d^{2}}{d x^{2}} \int_{-h}^{h} d x^{\prime} K\left(x-x^{\prime}\right) I\left(x^{\prime}\right) .
\end{aligned}
$$

Thus this term has been put in the form of a part of the first line of (B1). Similarly but more simply, the third term of (B1) is equal to the corresponding part of the first line, so that one has altogether

$$
\delta S[I]=2 \int_{-h}^{h} d x[\delta I(k, x)]\left(\frac{d^{2}}{d x^{2}}+k^{2}\right) \int_{-h}^{h} d x^{\prime} K\left(x-x^{\prime}\right) I\left(k, x^{\prime}\right),
$$

so that (3) implies that $\delta S[I]=0$.

\section{APPENDIX C \\ INTEGRATIONS FOR $I_{c}$ AND $I_{s}$}

Because $K\left(x-x^{\prime}\right)$ depends only on the difference between $x$ and $x^{\prime}$, it must be possible to rewrite the integrals (11) and (12) as single integrals. We do this without using the fact that $K$ is an even function of its argument. Define

$$
I_{e}\left(\alpha, \alpha^{\prime}\right)=\int_{-h}^{h} d x e^{i \alpha x} \int_{-h}^{h} d x^{\prime} K\left(x-x^{\prime}\right) e^{i \alpha^{\prime} x^{\prime}} .
$$

Changing integration variables $x \rightarrow-x^{\prime}$ and $x^{\prime} \rightarrow-x$ produces the relation

$$
I_{e}\left(-\alpha^{\prime},-\alpha\right)=I_{e}\left(\alpha, \alpha^{\prime}\right) \text {. }
$$


With this relation one expresses $I_{s}$ and $I_{c}$ as

$$
\begin{aligned}
& I_{s}=-\frac{1}{4}\left[2 I_{e}(\kappa, \kappa)-I_{e}(\kappa,-\kappa)-I_{e}(-\kappa, \kappa)\right], \\
& I_{c}=\frac{1}{4}\left[2 I_{e}(\kappa, \kappa)+I_{e}(\kappa,-\kappa)+I_{e}(-\kappa, \kappa)\right],
\end{aligned}
$$

so that we have

$$
\begin{aligned}
& I_{s}+I_{c}=\frac{1}{2}\left[I_{e}(\kappa,-\kappa)+I_{e}(-\kappa, \kappa)\right], \\
& I_{s}-I_{c}=-I_{e}(\kappa, \kappa) .
\end{aligned}
$$

For the reduction to single integrals we compute

$$
\begin{aligned}
I_{e}\left(\alpha, \alpha^{\prime}\right)= & \int_{-h}^{h} d x^{\prime} \int_{-h}^{h} d x e^{i \alpha x} e^{i \alpha^{\prime} x^{\prime}} K\left(x-x^{\prime}\right) \\
= & \int_{-h}^{h} d x^{\prime} \int_{-h-x^{\prime}}^{h-x^{\prime}} d y e^{i \alpha\left(y+x^{\prime}\right)} e^{i \alpha^{\prime} x^{\prime}} K(y) \\
= & \int_{-2 h}^{0} d y K(y) e^{i \alpha y} \int_{-h-y}^{h} d x^{\prime} e^{i\left(\alpha+\alpha^{\prime}\right) x^{\prime}}+\int_{0}^{2 h} d y K(y) e^{i \alpha y} \int_{-h}^{h-y} d x^{\prime} e^{i\left(\alpha+\alpha^{\prime}\right) x^{\prime}} \\
= & \frac{1}{i\left(\alpha+\alpha^{\prime}\right)}\left[\int_{-2 h}^{0} d y K(y) e^{i \alpha y}\left(e^{i\left(\alpha+\alpha^{\prime}\right) h}-e^{-i\left(\alpha+\alpha^{\prime}\right)(h+y)}\right)\right. \\
& \left.+\int_{0}^{2 h} d y K(y) e^{i \alpha y}\left(e^{i\left(\alpha+\alpha^{\prime}\right)(h-y)}-e^{-i\left(\alpha+\alpha^{\prime}\right) h}\right)\right] \\
= & \frac{1}{i\left(\alpha+\alpha^{\prime}\right)}\left[\int_{-2 h}^{0} d y K(y)\left(e^{i\left(\alpha+\alpha^{\prime}\right) h} e^{i \alpha y}-e^{-i\left(\alpha+\alpha^{\prime}\right) h} e^{-i \alpha^{\prime} y}\right)\right. \\
& \left.+\int_{0}^{2 h} d y K(y)\left(e^{i\left(\alpha+\alpha^{\prime}\right) h} e^{-i \alpha^{\prime} y}-e^{-i\left(\alpha+\alpha^{\prime}\right) h} e^{i \alpha y}\right)\right] .
\end{aligned}
$$

The case $\alpha^{\prime}=-\alpha$ is worked out directly to show

$$
I_{e}(\alpha,-\alpha)=\int_{-2 h}^{0} d y K(y)(2 h+y) e^{i \alpha y}+\int_{0}^{2 h} d y K(y)(2 h-y) e^{i \alpha y}
$$

Substitution of (C7) and (C8) into (C5) and (C6) yields

$$
\begin{aligned}
& I_{s}+I_{c}=\int_{-2 h}^{0} d y K(y)(2 h+y) \cos \kappa_{n} y+\int_{0}^{2 h} d y K(y)(2 h-y) \cos \kappa_{n} y \\
& I_{s}-I_{c}=-\frac{1}{\kappa_{n}}\left[\int_{-2 h}^{0} d y K(y) \sin \left[\kappa_{n}(2 h+y)\right]+\int_{0}^{2 h} d y K(y) \sin \left[\kappa_{n}(2 h-y)\right]\right]
\end{aligned}
$$

In the special case, which we have here, in which $K(y)=K(-y)$ these simplify slightly to

$$
\begin{aligned}
& I_{s}+I_{c}=2 \int_{0}^{2 h} d y K(y)(2 h-y) \cos \kappa_{n} y, \\
& I_{s}-I_{c}=-\frac{2}{\kappa_{n}} \int_{0}^{2 h} d y K(y) \sin \left[\kappa_{n}(2 h-y)\right]
\end{aligned}
$$

which are the single integrals that we wanted to obtain. 


\section{APPENDIX D \\ EVALUATION OF $I_{s}+I_{c}$}

Now we evaluate the sum $I_{s}+I_{c}$, starting by writing

$$
I_{s}+I_{c}=I_{1}+I_{2}
$$

where

$$
\begin{aligned}
& I_{1} \stackrel{\text { def }}{=} 4 h \int_{0}^{2 h} d y \frac{e^{i k \sqrt{y^{2}+a^{2}}}}{\sqrt{y^{2}+a^{2}}} \cos \kappa_{n} y, \\
& I_{2} \stackrel{\text { def }}{=}-2 \int_{0}^{2 h} d y \frac{e^{i k \sqrt{y^{2}+a^{2}}}}{\sqrt{y^{2}+a^{2}}} y \cos \kappa_{n} y .
\end{aligned}
$$

\section{A. Evaluation of the integral $I_{2}$}

In $I_{2}, a$ can be set to zero to obtain

$$
\begin{aligned}
I_{2} & \approx-2 \int_{0}^{2 h} d y e^{i k y}\left(e^{i \kappa_{n} y}+e^{i \kappa_{n} y}\right) \\
& =\frac{i}{k+\kappa_{n}}\left[e^{i 2\left(k+\kappa_{n}\right) h}-1\right]+\frac{i}{k-\kappa_{n}}\left[e^{i 2\left(k-\kappa_{n}\right) h}-1\right] .
\end{aligned}
$$

Of note is the appearance (in the denominator of the second term) of the difference $k-\kappa_{n}$, which is small (and complex). We are interested in the case that $\left|k-\kappa_{n}\right| \ll \kappa_{n}$ but $2 h\left|k-\kappa_{n}\right|$ may or may not be small. Very roughly, we expect $\left|k-\kappa_{n}\right| / \kappa_{n}$ to be of the order of $1 / 2 \ln (2 h / a)$, which is perhaps 0.05 . For the fifth resonance, $\kappa_{5}=5 \pi /(2 h)$, leading to $2 h\left|k-\kappa_{n}\right|=(0.05) 5 \pi \sim 0.8$.

\section{B. Evaluation of the integral $I_{1}$}

We want to evaluate

$$
I_{1} \stackrel{\text { def }}{=} 4 h \int_{0}^{2 h} d y \frac{e^{i k \sqrt{y^{2}+a^{2}}}}{\sqrt{y^{2}+a^{2}}} \cos \kappa_{n} y
$$

under the conditions that $a$ satisfies $\kappa_{n} a \ll 1$ and $a / h \ll 1$, and we are interested in the case that $\left|k-\kappa_{n}\right| / \kappa_{n} \ll 1$ but $2 h\left|k-\kappa_{n}\right| \kappa_{n}$ may or may not be small. In these circumstances we have

$$
I_{1} \approx 4 h \int_{0}^{2 h} d y \frac{e^{i k y}}{\sqrt{y^{2}+a^{2}}} \cos \kappa_{n} y=4 h\left[I_{1}^{(1)}-I_{1}^{(2)}\right],
$$

where we define

$$
\begin{aligned}
& I_{1}^{(1)} \stackrel{\text { def }}{=} \int_{0}^{2 h} d y \frac{1}{\sqrt{y^{2}+a^{2}}}=\sinh ^{-1}(2 h / a) \approx \ln (4 h / a), \\
& I_{1}^{(2)} \stackrel{\text { def }}{=} \int_{0}^{2 h} d y \frac{1}{\sqrt{y^{2}+a^{2}}}\left[1-e^{i k y} \cos \kappa_{n} y\right] \approx \int_{0}^{2 h} d y \frac{1}{y}\left[1-e^{i k y} \cos \kappa_{n} y\right],
\end{aligned}
$$

which implies

$$
I_{1} \approx 4 h\left[\ln \frac{4 h}{a}-\int_{0}^{2 h} \frac{d y}{y}\left(1-e^{i k y} \cos \kappa_{n} y\right)\right] .
$$

Adding (D9) and (D4) then yields (19). 


\section{REFERENCES}

[1] R. W. P. King, The Theory of Linear Antennas. Cambridge, MA: Harvard Univ. Press, 1956.

[2] F. M. Tesche, "On the analysis of scattering and antenna problems using the singularity expansion technique," IEEE Trans. Antennas Propag., vol. AP-21, pp. 53-62, Jan. 1973.

[3] C. E. Baum, "Emerging technology for transient and broad-band analysis and synthesis of antennas and scatterers," Proc. IEEE, vol. 64, pp. 1598-1616, Nov. 1976; C. E. Baum, "The singularity expansion method," in Transient Electromagnetic Fields, L. B. Felsen, Ed. New York: SpringerVerlag, 1975. 